\title{
Optimal Outsourcing for Intellectual Property Protection and Production Cost Minimization
}

\author{
John P Kim \\ Dept. of Mechanical Engineering. \\ University of Michigan \\ Ann Arbor, MI 48109-2125, USA \\ johnpk@umich.edu
}

\author{
Karim Hamza \\ Dept. of Mechanical Engineering. \\ University of Michigan \\ Ann Arbor, MI 48109-2125, USA \\ khamza@umich.edu
}

\author{
Kazuhiro Saitou \\ Dept. of Mechanical Engineering. \\ University of Michigan \\ Ann Arbor, MI 48109-2125, USA \\ kazu@umich.edu
}

\begin{abstract}
This paper presents a methodology for optimal outsourcing of products. Outsourcing of products can have the advantage of reducing the production cost, but often causes a risk that important technology may leak and get used by competitors. To help reduce the risk of intellectual property (IP) leakage, a model proposed in this paper assumes that it is possible to separate some of the important geometrical features on some of the product parts that are outsourced, and then manufacture them in-house. The model estimates the fraction of IP-value that is subject to risk of leakage based on patent claims and how they relate to the outsourced parts and/or features. Production cost is modelled by assuming a base cost for manufacturing parts in-house, and then a discount rate is applied if the decision to outsource is made. Separation of geometrical features from manufactured parts introduces additional cost, which is modelled as an overhead if the decision to separate features is made. The outsourcing management process is then viewed as a two-objective problem, with the objectives being the minimization of both the fraction of IP-value at risk of leakage, as well as the production cost. A case study of an auto-slide-hinge mechanism is presented, in which the two-objective optimization problem is transformed into a singleobjective constrained problem. Genetic algorithm is then applied iteratively on the problem in order generate the Pareto-plot that visualizes the trade-offs between the two objectives.
\end{abstract}

Index Terms - Intellectual property protection, Outsourcing management, Genetic Algorithm.

\section{INTRODUCTION}

Outsourcing is a practice often used by companies nowadays to reduce the in-house production costs, which aims at maximizing their profitability and maintaining a competitive edge. Maintaining a competitive edge can be greatly undermined however if a company's intellectual property (IP) is leaked out, and its products are imitated by competitors. Leakage of IP can happen internally from within the company itself, or externally through suppliers that manufacture parts of the company's products. Preventive measures for inhibiting internal IP leakage are fairly well established. Common practices include: e-mail, instant messaging and network protection [1-4], role-based data access [5], as well as researcher tracing systems [6].
Prevention of external IP leakage while taking advantage of outsourcing to reduce manufacturing costs is not an easy task. The main difficulty is the lack of control [7] on the IP security at the supplier end.

Recovery of IP value is generally not impossible after a leakage occurs. Patents help protect IP. In the event of discovery and proof of unlawful imitation of a company's product, the company could file a lawsuit that entitles it to compensation. An example is the die-casting machine of T-company [8], which has a pretty unique design developed in the 1980s, still has a decent market possession despite imitation. Proving that unlawful imitation had occurred can have varying levels of difficulty depending on the product type. Watermarking technologies $[9,10]$ are used extensively in audio and video digital multimedia products. Special forms of watermarking have been proposed for three-dimensional geometry computer aided design (CAD) models [11-14], and thermal tags [15] have been proposed for electronic chips. Mechanical hardware products however, are possibly the most difficult to prove whether a similar product by a competitor is truly an unlawful imitation or not. The issue is further complicated in product like cell phones that have a very short lifecycle. This is because the product lifecycle of such products can be shorter than the duration of a lawsuit [16]. Hence for such products, it is of great importance to prevent the IP leakage during the product lifecycle.

Reducing the risk of IP leakage and reducing the cost of manufacturing via outsourcing can be a tough decision to make. A number of articles in the literature [7, 17-19] have viewed outsourcing as a binary decision. In this paper, partial-outsourcing is considered, where some features of parts of a product can be separated thereby allowing outsourcing of the base part and some of the features, while using in-house manufacturing for the more important features and the final assembly. Outsourcing is posed as a multi-objective optimization problem of reducing the IPvalue at risk of leakage and the manufacturing cost.

This paper started with a motivation and brief review of relevant work in the literature, which concludes to the importance of the research conducted in this paper. The next section explains the modelling details of the IP leakage risk and manufacturing cost functions. A case study of an auto-slide hinge mechanism is then presented to demonstrate the proposed method. The paper then concludes with a discussion and future work. 


\section{METHOD}

The model for risk of IP leakage and manufacturing cost assume a product that is composed of a number of parts, and that each part can have one or several features. The model also assumes the existence of patent documentation, draft or approved, that defines claims about parts and features. Consider an example hypothetical garden-care product consisting of two parts, which is simplified as in Fig. 1, and has three patent claims listed as:

- Claim 1: Part 1 attaches to a regular lawn-mowing machine

- Claim 2: Part 2 performs a weed-disinfection operation while regular lawn-mowing is in process

- Claim 3: A pin-feature in Part 2 has a special docking mechanism in a hole-feature in Part 1 that allows for safe operation, as well as easy detachment.

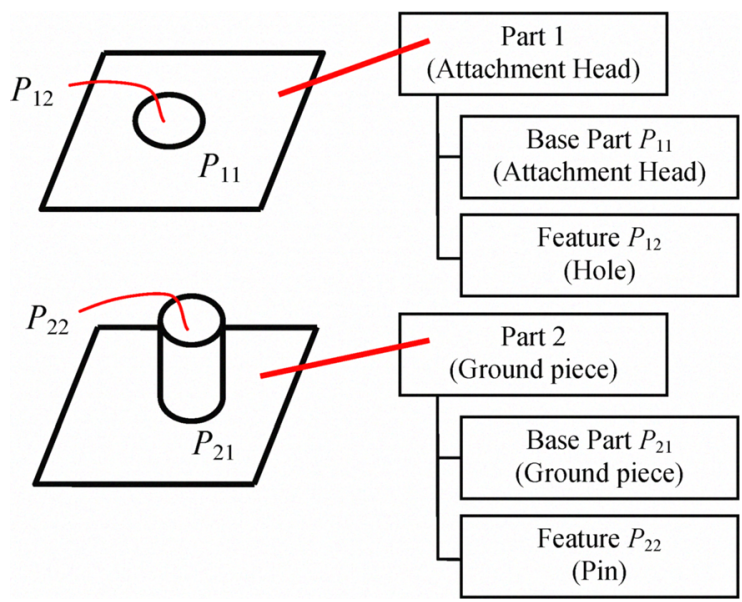

Fig. 1 Simplified model of a garden-care product

An example of partial outsourcing through feature separation is illustrated in Fig. 2. In this outsourcing scenario, the base part of the attachment head $\left(P_{11}\right)$ is outsourced, but the hole-feature $\left(P_{12}\right)$ is manufactured inhouse at a later stage. This outsourcing scenario takes advantage of reduced manufacturing of the base part without putting all of the IP-value associated with the part at the risk of leakage.

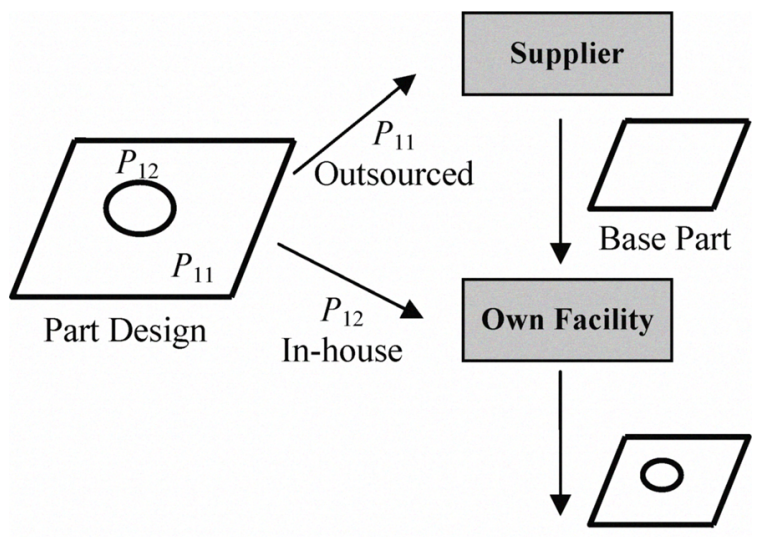

Full Part

Fig. 2 Illustration of a partial outsourcing scenario

The following subsections provide the details about the optimal outsourcing model. The model makes the following assumptions:

- Outsourcing a part or feature puts the IP value in it at a $100 \%$ risk of being leaked out, while manufacturing it within the company's own facilities (insourcing) is $100 \%$ secure.

- Only one supplier is available for outsourcing.

- Outsourcing parts or features reduces the manufacturing cost at a certain rate. This rate is the same for all the parts and features if they are outsourced.

- Separating features in a part such that they are manufactured at a different location than the base part incurs a cost penalty. This cost penalty can be different, depending on the feature in question.

- The model user is able to estimate the relative importance of the IP-value of the patent claims as weighting factors.

- The model user is able to estimate the manufacturing cost of every part and feature, as a fraction of the total cost of the product if it were to be manufactured inhouse.

\section{A. Model Parameters and Design Variables}

In this paper, every part and/or feature is denoted as $P_{i j}$ (Fig. 1), where:

$i \quad$ is an index on the part number. $i \in\{1, \ldots, n\}$

$n \quad$ is the number of parts in the product

$j \quad$ is an index on the feature on a part number. $j \in$ $\left\{1, \ldots, r_{i}+1\right\}$. As a notation, $j=1$ indicates the base part, while $j>1$ indicates features on the base part

$r_{i} \quad$ is the number of features on part $i$

$k \quad$ is an index on the patent claims. $k \in\{1, \ldots, m\}$

$m$ is the number of claims in the patent

In the simple garden-care product in Fig. 1, there are two parts $(n=2)$, one additional feature on each part $\left(r_{1}=\right.$ $\left.r_{2}=1\right)$, and three claims $(m=3)$.

Based on the wording of the patent claims, one can define a claim to part/feature relationship matrix $S=\left\{s_{i j k}\right\}$ via the following rules:

- $s_{i j k}=1$ for $j>1$, if feature $j$ in part $i$ is mentioned in claim $k$

- $s_{i 1 k}=1$ if the base part $i$ is mentioned in claim $k$ as performing a certain functionality other than simply "containing" features that perform functionality.

- $s_{i j k}=0$ otherwise

In the garden-care product in Fig. 1, based on the listed patent claims, the resulting $S$ matrix is shown in Table 1 . Explanation of how the claims are used to construct the $S$ matrix is done as follows:

- Claim 1 mentions functionality of part 1 , so $s_{111}=1$.

- Claim 2 mentions functionality of part 2 , so $s_{212}=1$. 
TABLE $1 S$ matrix for the garden care product

\begin{tabular}{|l|c|c|c|}
\hline & Claim 1 & Claim 2 & Claim 3 \\
\hline$P_{11}$ & 1 & 0 & 0 \\
\hline$P_{12}$ & 0 & 0 & 1 \\
\hline$P_{21}$ & 0 & 1 & 0 \\
\hline$P_{22}$ & 0 & 0 & 1 \\
\hline
\end{tabular}

- Claim 3 mentions features $P_{12}$ and $P_{22}$, so $s_{123}=s_{233}=$ 1. Base parts $P_{11}$ and $P_{21}$ do not play any roles in the functionality mentioned in the claim, thus $s_{113}=s_{213}=0$

- All other values in the $\mathrm{S}$ matrix are zero.

The design variables are denoted as $x_{i j}$, where:

$x_{i j}=\left\{\begin{array}{lc}1 & \text { if the manufacturing of feature } j \text { is outsourced } \\ 0 & \text { if the manufacturing of feature } j \text { is not outsourced }\end{array}\right.$

The next subsections explain the details of the IP-value at risk of leakage and the manufacturing cost functions.

\section{B. Objective Functions}

The outsourcing management decisions are posed as a two-objective optimization problem as:

$$
\text { Minimize } \quad f_{1}, f_{2}
$$

where:

$f_{1} \quad$ is an objective indicating the IP-value at risk of leakage

$f_{2} \quad$ is an objective indicating the manufacturing cost of the product

The objective for IP-value at risk leakage is defined as:

$$
f_{1}=\sum_{k=1}^{m} w_{k} \frac{\sum_{i=1}^{n} \sum_{j=1}^{r_{i}} s_{i j k} x_{i j}}{\sum_{i=1}^{n} \sum_{j=1}^{r_{i}} s_{i j k}}
$$

where $w_{k}$ is the IP-value weighting factor associated with claim $k$ that the model user assigns. $w_{k}>0$. In this paper, $w_{k}$ is chosen such that:

$$
\sum_{k=1}^{m} w_{k}=1
$$

With such choice of $w_{k}$, the value of $f_{1}$ will simply be the fraction of total IP-value that is exposed to high risk of leakage. $f_{1}=1$ will correspond to all parts and features being outsourced (hence all the IP-value is at risk), while $f_{1}$ $=0$ will correspond to all parts and features being insourced (hence no IP-value is at risk)

The manufacturing cost objective is defined as:

$$
\begin{aligned}
f_{2} & =\sum_{x_{i j}=x_{i 1}} c_{i j}\left[\beta x_{i j}+\left(1-x_{i j}\right)\right] \\
& +\sum_{x_{i j} \neq x_{i 1}} \gamma_{i j} c_{i j}\left[\beta x_{i j}+\left(1-x_{i j}\right)\right]
\end{aligned}
$$

$c_{i j} \quad$ is the fraction of the total manufacturing cost of the product that is allocated to feature $j$ of part $i$. $c_{i j}>0$, and by definition:

$$
\sum_{i=1}^{n} \sum_{j=1}^{r_{i}} c_{i j}=1
$$

$\beta \quad$ is the discount rate at which the manufacturing cost of a part or feature is reduced, when it is outsourced. This should also account for shipping and handling of parts from the supplier.

$\gamma_{i j} \quad$ is a cost penalty incurred when decision is made to separate the manufacturing of a feature from base part (i.e. when $\left.x_{i j} \neq x_{i 1}\right), \gamma_{i j}>1$ for $j>1$. This can also account for additional setup and tooling.

\section{Sample Results}

Considering the garden-care product in Fig. 1, the manufacturing discount rate for outsourcing is assumed to be $50 \%$ off $(\beta=0.5)$. The weighting factors for the IPvalue in each of the patent claims $\left(w_{k}\right)$ is listed in Table 2 , while the fractions of total manufacturing cost $\left(c_{i j}\right)$ and feature separation penalty $\left(\gamma_{i j}\right)$ are listed in Table 3 .

Since there are only 4 design variables $\left(x_{11}, x_{12}, x_{21}, x_{22}\right)$ in this example, the total number of possible outsourcing scenarios is $2^{4}=16$. This allows for generation and examination of all of them (exhaustive search) in order to generate the Pareto-plot in Fig. 3, which shows the tradeoff between fraction IP-value at risk of leakage $\left(f_{1}\right)$ and the total cost of manufacturing $\left(f_{2}\right)$. Listing of all the outsourcing scenarios is provided in Table 4.

TABLE 2 IP-value weighting factors for the garden care product

\begin{tabular}{|l|r|}
\hline & \multicolumn{1}{|c|}{$w_{k}$} \\
\hline Claim 1 & 0.25 \\
\hline Claim 2 & 0.15 \\
\hline Claim 3 & 0.60 \\
\hline
\end{tabular}

TABLE 3 Cost and feature separation factors for the garden care product

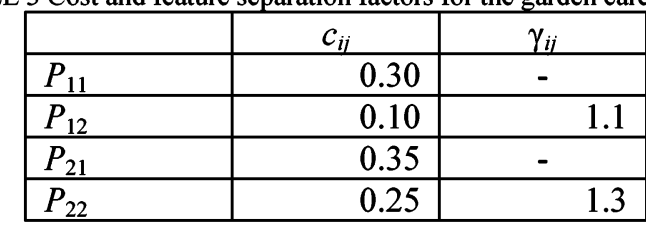

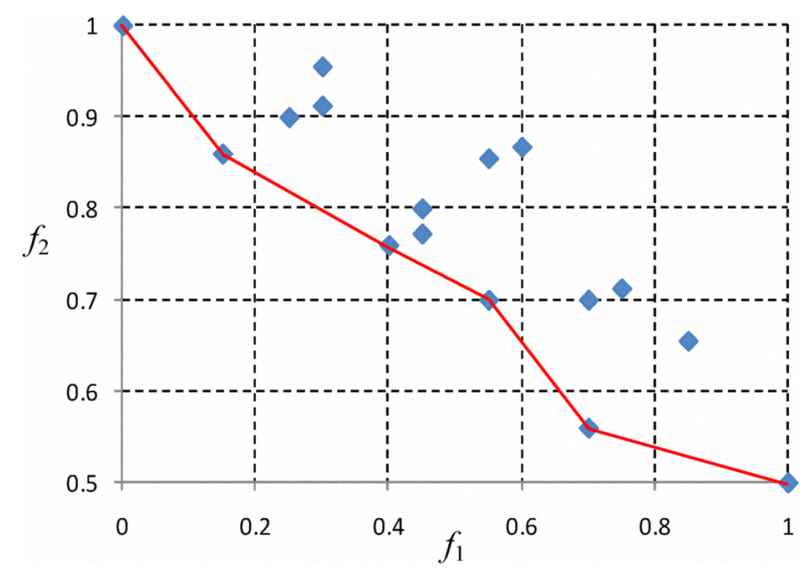

Fig. 3 Outsourcing scenarios of the garden care product 
TABLE 4 Outsourcing scenarios for the garden care product

\begin{tabular}{|c|c|c|c|c|c|c|}
\hline Scenario & $x_{11}$ & $x_{12}$ & $x_{21}$ & $x_{22}$ & $f_{1}$ & $f_{2}$ \\
\hline $1^{*}$ & 0 & 0 & 0 & 0 & 0.000 & 1.000 \\
\hline $2^{*}$ & 1 & 0 & 0 & 0 & 0.150 & 0.860 \\
\hline 3 & 0 & 1 & 0 & 0 & 0.300 & 0.955 \\
\hline 4 & 1 & 1 & 0 & 0 & 0.450 & 0.800 \\
\hline 5 & 0 & 0 & 1 & 0 & 0.250 & 0.900 \\
\hline $6^{*}$ & 1 & 0 & 1 & 0 & 0.400 & 0.760 \\
\hline 7 & 0 & 1 & 1 & 0 & 0.550 & 0.855 \\
\hline 8 & 1 & 1 & 1 & 0 & 0.700 & 0.700 \\
\hline 9 & 0 & 0 & 0 & 1 & 0.300 & 0.913 \\
\hline 10 & 1 & 0 & 0 & 1 & 0.450 & 0.773 \\
\hline 11 & 0 & 1 & 0 & 1 & 0.600 & 0.868 \\
\hline 12 & 1 & 1 & 0 & 1 & 0.750 & 0.713 \\
\hline $13^{*}$ & 0 & 0 & 1 & 1 & 0.550 & 0.700 \\
\hline $14^{*}$ & 1 & 0 & 1 & 1 & 0.700 & 0.560 \\
\hline 15 & 0 & 1 & 1 & 1 & 0.850 & 0.655 \\
\hline $16 *$ & 1 & 1 & 1 & 1 & 1.000 & 0.500 \\
\hline
\end{tabular}

It should be noted that when considering products of realistic level of complexity, the total number of all possible scenarios is $O\left(2^{n} \times \max (r)\right)$, which can quickly become too large. However, in practice, only the Paretooptimal scenarios are of interest. One popular method for generating Pareto-optimal solutions for multi-objective optimization problems is genetic algorithm (GA), which is used in the next section. The next section presents a case study of a realistic product in order to further demonstrate the proposed approach.

\section{CASE STUDY}

\section{A. Problem Data}

This case study considers the three main parts of a semiautomated slide hinge mechanism manufactured by LGcompany and used in several cell phone models (Fig. 4). The design is patented $[20,21]$ and has six claims that mention the considered parts and features. Summary of the claims is listed as:

- Claim 1: a slide part includes a housing $\left(P_{11}\right)$ in which a setting hole is formed in a back surface of the housing, and a slide guide $\left(P_{21}\right)$ sliding along the guide bar mounted on the body part.

- Claim 2: a cover plate $\left(P_{31}\right)$ combines with the inside of the housing $\left(P_{11}\right)$ to cover the setting hole.

- Claim 3: a guide locking protrusion $\left(P_{22}\right)$ is formed on an outside surface of the slide guide; and a guide locking projection $\left(P_{13}\right)$ is formed on an inside surface in the housing. $P_{13}$ and $P_{22}$ lock together.

- Claim 4: a guide pin $\left(P_{33}\right)$ is formed on a surface of the cover plate mates with a guide hole $\left(P_{12}\right)$ formed so that the guide pin may be contained on another surface from among mutual contact surfaces of the housing $\left(P_{11}\right)$.

- Claim 5: a passage hole $\left(P_{32}\right)$ is formed in the cover plate so that a flexible printed circuit board electrically connecting the body part with the slide part may pass.
- Claim 6: the slide guide $\left(P_{21}\right)$ is includes a lubricant material.

The claims in the patent are used to construct the $S$ matrix in accordance with the rules in section II. The $S$ matrix is listed in Table 5.

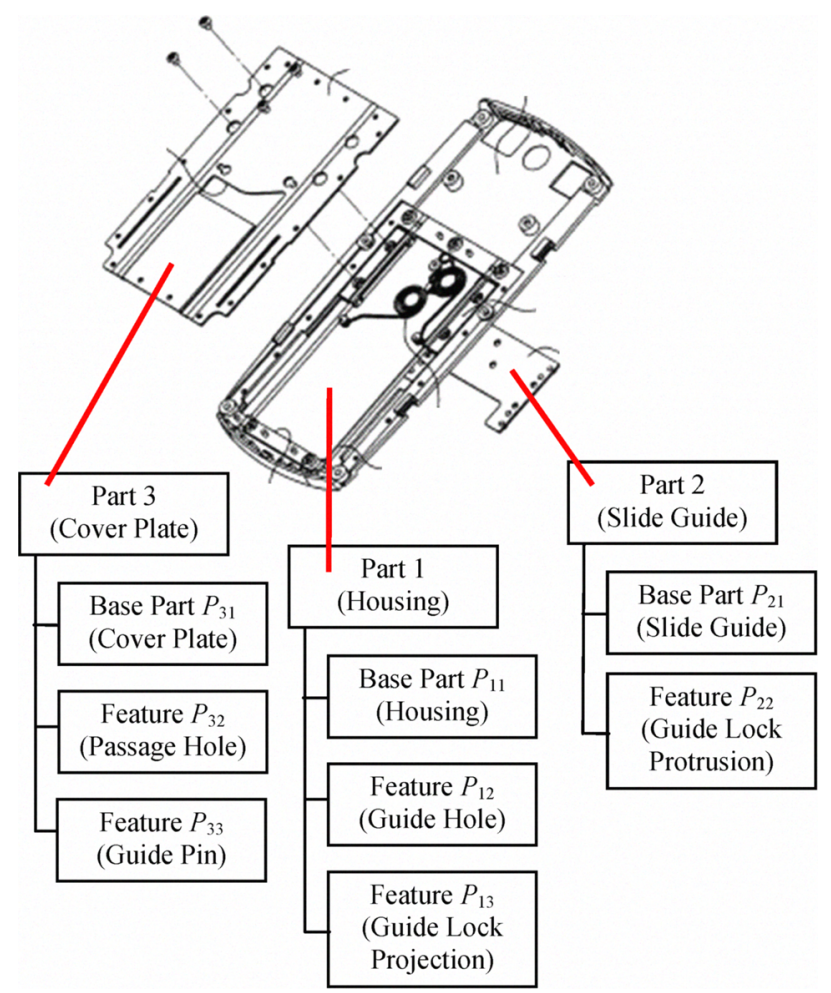

Fig. 4 Main parts and features of a semi-auto slide hinge mechanism

TABLE $5 S$ matrix for the case study product

\begin{tabular}{|c|c|c|c|c|c|c|}
\hline & \multicolumn{7}{|c|}{ Claim $k$} \\
\cline { 2 - 7 } & 1 & 2 & 3 & 4 & 5 & 6 \\
\hline$P_{11}$ & 1 & 1 & 0 & 1 & 0 & 0 \\
\hline$P_{12}$ & 0 & 0 & 0 & 1 & 0 & 0 \\
\hline$P_{13}$ & 0 & 0 & 1 & 0 & 0 & 0 \\
\hline$P_{21}$ & 1 & 0 & 0 & 0 & 0 & 1 \\
\hline$P_{22}$ & 0 & 0 & 1 & 0 & 0 & 0 \\
\hline$P_{31}$ & 0 & 1 & 0 & 0 & 0 & 0 \\
\hline$P_{32}$ & 0 & 0 & 0 & 0 & 1 & 0 \\
\hline$P_{33}$ & 0 & 0 & 0 & 1 & 0 & 0 \\
\hline
\end{tabular}

IP-value weights for the claims are listed in Table 6, while the cost and feature separation factors are listed in Table 7. The manufacturing cost discount when outsourcing was assumed to be $50 \%$ off $(\beta=0.5)$.

TABLE 6 IP-value weighting factors for the case study product

\begin{tabular}{|l|r|}
\hline & $w_{k}$ \\
\hline Claim 1 & 0.097 \\
\hline Claim 2 & 0.150 \\
\hline Claim 3 & 0.332 \\
\hline Claim 4 & 0.235 \\
\hline Claim 5 & 0.142 \\
\hline Claim 6 & 0.044 \\
\hline
\end{tabular}


TABLE 7 Cost and feature separation factors for the case study product

\begin{tabular}{|l|r|r|}
\hline & $c_{i j}$ & $\gamma_{i j}$ \\
\hline$P_{11}$ & 0.267 & - \\
\hline$P_{12}$ & 0.045 & 1.1 \\
\hline$P_{13}$ & 0.111 & \\
\hline$P_{21}$ & 0.142 & - \\
\hline$P_{22}$ & 0.053 & 1.2 \\
\hline$P_{31}$ & 0.244 & - \\
\hline$P_{32}$ & 0.049 & 1.1 \\
\hline$P_{33}$ & 0.089 & 1.3 \\
\hline
\end{tabular}

\section{B. Results and Discussion}

Optimization is employed in order to generate the Pareto-optimal outsourcing scenarios. The simple example in section II demonstrated that can be non-convex regions in the Pareto-plot (Fig. 3), which in turn means that combining the two objectives $f_{1}, f_{2}$ into a single objective via weighting then conduct optimization by varying the weights will not be able to generate all the Pareto-optimal scenarios [22]. Instead, the problem is re-posed as a single objective constrained problem as follows:

$$
\begin{array}{ll}
\text { Minimize } & f_{1} \\
\text { S.T. } & f_{2} \leq F
\end{array}
$$

where:

$F \quad$ is the constraint level on $f_{2}$, which is varied between $\beta$ (converges to everything being outsourced) and 1 (converges to nothing to being outsourced). The step variation on $F$ can be adjusted to allow for the discovery of most of the Pareto-optimal scenarios.

A simple binary-coded genetic algorithm (GA) [23] is employed to the solve the optimization problem in equations $(6,7)$ for different values of $F$ in order to generate the Pareto-optimal scenarios. Tuning parameters of the optimization runs are listed in Table 8. The discovered Pareto-optimal outsourcing scenarios are plotted in Fig. 5. Since the number of design variables in this case study is only 8 , the total number of possible outsourcing scenarios is only $2^{8}=256$, which is a small enough number that they can all be explored. A plot of all the possible outsourcing scenarios is shown in Fig. 6 . Comparison of Fig. 5 and Fig. 6 shows that the GA was successful in discovering all the Pareto-optimal outsourcing scenarios.

TABLE 8 Tuning parameters for the optimization algorithm

\begin{tabular}{|l|r|}
\hline \multicolumn{1}{|c|}{ Parameter } & Value \\
\hline$F$ minimum value & 0.500 \\
\hline$F$ maximum value & 1.000 \\
\hline$F$ step & 0.002 \\
\hline GA population size & 50 \\
\hline GA number of generations & 20 \\
\hline GA crossover type & Binary string \\
\hline GA crossover probability & 0.85 \\
\hline GA bit mutation probability & 0.05 \\
\hline
\end{tabular}

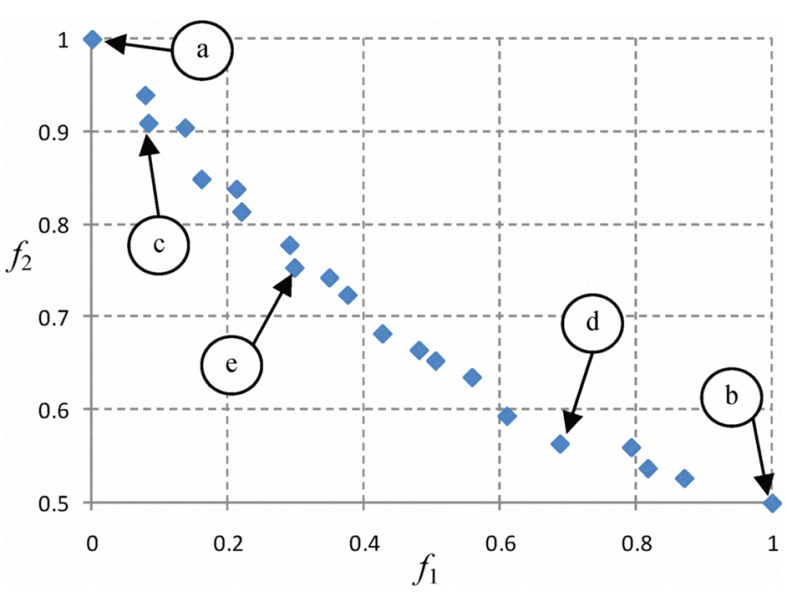

Fig. 5 Pareto-optimal outsourcing scenarios for the case study product

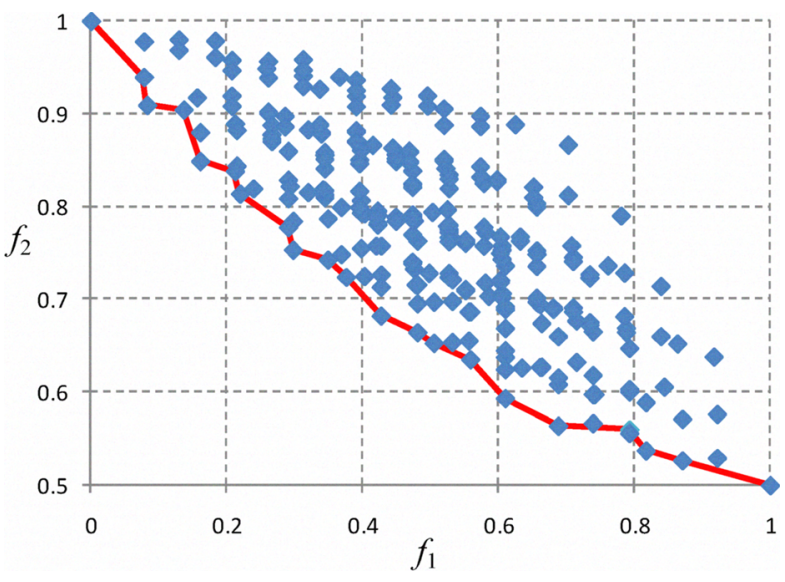

Fig. 6 All outsourcing scenarios for the case study product

Some note-worthy Pareto-optimal outsourcing scenarios are selected from Fig. 4 . The values of the design variables and objective functions of these scenarios are listed in Table 9. Summary of the scenarios are discussed as follows:

- Scenario a: represents that case when all parts and features are being manufactured in-house, which gives the minimum risk of IP leakage, but maximal production cost.

- Scenario b: represents that case when all parts and features are outsourced, which gives the minimum production cost, but all the IP-value is at a risk of leakage.

- Scenario c: represents a conservative case of protecting the IP-value by only outsourcing a small portion of the product, which is the base part of the Cover Plate $\left(P_{31}\right)$. This results in an appreciable reduction of the manufacturing cost.

- Scenario d: represents a case where most of the product is being outsourced, yet by only withholding two critical features from outsourcing $\left(P_{12}\right.$ and $\left.P_{22}\right)$, the IPvalue that's at risk of leakage is reduced by $31.2 \%$ while only increasing the production cost by $6.4 \%$ compared to scenario $b$ 
- Scenario e: represents a balanced case between putting the IP-value at risk verses reducing the manufacturing cost. In this case, only the base parts $\left(P_{11}, P_{21}\right.$ and $\left.P_{31}\right)$ are being outsourced, while the additional features are manufactured in-house.

TABLE 9 Select Pareto-optimal outsourcing scenario

\begin{tabular}{|c|c|c|c|c|c|}
\hline & \multicolumn{5}{|c|}{ Scenario } \\
\cline { 2 - 6 } & $\mathrm{a}$ & $\mathrm{b}$ & $\mathrm{c}$ & $\mathrm{d}$ & $\mathrm{e}$ \\
\hline$x_{11}$ & 0 & 1 & 0 & 1 & 1 \\
\hline$x_{12}$ & 0 & 1 & 0 & 0 & 0 \\
\hline$x_{13}$ & 0 & 1 & 0 & 1 & 0 \\
\hline$x_{21}$ & 0 & 1 & 0 & 1 & 1 \\
\hline$x_{22}$ & 0 & 1 & 0 & 0 & 0 \\
\hline$x_{31}$ & 0 & 1 & 1 & 1 & 1 \\
\hline$x_{31}$ & 0 & 1 & 0 & 1 & 0 \\
\hline$x_{31}$ & 0 & 1 & 0 & 1 & 0 \\
\hline$f_{1}$ & 0.000 & 1.000 & 0.083 & 0.688 & 0.298 \\
\hline$f_{2}$ & 1.000 & 0.500 & 0.909 & 0.564 & 0.753 \\
\hline
\end{tabular}

\section{SUMMARY AND FUTURE WORK}

This paper presented a modelling approach to outsourcing decisions that promotes partial outsourcing in order to reduce the manufacturing cost without significantly compromising the IP-value by putting it at risk of leakage to competitors. The outsourcing problem is viewed as a multi-objective problem, for which, genetic algorithms seem to be effective at generating the Paretooptimal scenarios for outsourcing.

Future work would include more complex products and case studies, as well as using multi-objective genetic algorithms [22] for direct generation of the Pareto-optimal solutions without the need for the single-objective constrained formulation discussed in section III.

Future extensions of the modelling could also encompass:

- Semi-automated generation of the $S$ matrix from patent documentation based on natural language processing [24].

- Semi-automated generation of the $S$ matrix from similar product models.

- Probabilistic modelling of IP risk of leakage, which while more complex, is more realistic than assuming that outsourcing will always result in IP leakage.

- Part and product quality as a third objective in conjunction with cost and IP protection.

- Multiple suppliers to choose from, which can have different manufacturing cost discount rates, different levels of risk of IP leakage and manufacturing quality capabilities.

- Alternative design materials, manufacturing and assembly processes as part of the design variables.

\section{REFERENCES}

[1] Marshall Fisher, Ananth Ramen, "Managing short life-cycle products," Ascet., Vol. 1, Jan. 1999.

[2] James Blake, "The critical role of data loss prevention in governance," A MIMECast white paper, Nov. 2008.

[3] Jennifer deJong, Kevin Beaver, "Six steps to reduce risk and improve control over real-time communications," Quest software white paper, Apr. 2008.

[4] Mark Mizrahi, "Network access protection," Global Knoeledge expert reference series of white paper, Sep. 2006

[5] Wang, Y., Ajoku, P., Brustoloni, J. and Nnaji, B., "Intellectual Property Protection in Collaborative Design through Lean Information Modeling and Sharing," J. of Computer Science and Engineering, Vol. 6, pp. 149-159, June 2006.

[6] S. Srinivasa Rao, E G Rajan, K Lalkishore, "Human activity tracking using RFID tags", Int. J. of Computer Science and Network Security, Vol. 9, No.1 , 2009

[7] John Sterlicchi, "Manufacturing IP Securely," Information Security, Vol. 5, No. 7, pp. 31-33, 2008.

[8] Alexandre A. Pollak, Carl Thibault, Alain Bourbonnais, Richard Laveau, "Multiple-Slide Die-Casting System ," United States patent $6938671,2005$.

[9] Cox, I. J., Kilian, J., Leightont, T., and Shamoon, T., "Secure Spread Spectrum Watermarking for Images, Audio and Video," IEEE Trans. Image Process., Vol. 6, No. 2, pp. 1673-1687, 1997.

[10] Barni, M., and Barolini, F., "Data Hiding for Fighting Piracy," IEEE Signal Process. Mag., Vol. 21, No. 2, pp. 28-39, 2004.

[11] Ohbuchi, R., Masuda, H., and Aono, M., "Watermarking ThreeDimensional Polygonal Models through Geometric and Topological Modifications," IEEE J. Sel. Areas Commun., Vol. 16, No. 4, pp. 551-560, 1998

[12] Harte, T., and Bors, A. G., "Watermarking Graphical Objects," IEEE Proceedings of the 14th International Conference on Digital Signal Processing, Vol. 2, pp. 709-721, 2002.

[13] Ohbuchi, R., Masuda, H., and Aono, M., 1999, "A Shape-Preserving Data Embedding Algorithm for NURBS Curves and Surfaces," Proceedings of the IEEE International Conference on Computer Graphics, Canmore, Canada, pp. 180-187, 1999.

[14] Ko, K.H., Maekawa, T. and Patrikalakis, N.M., "An algorithm for optimal free-form object matching," Computer Aided Design, Vol. 35, pp. 913-923, 2003.

[15] Marsh, C.; Kean, T. and McLaren, D., "Protecting designs with a passive thermal tag," IEEE International Conference on Electronics, Circuits and Systems, pp. 218-221, 2008.

[16] James D. Adams, J.Roger Clemmons, Paula E. Stephan, "How rapidly does science leak out," Rensselaer, Working papers in economics, Jan. 2006.

[17] Grinols, E. and Lin, H.C., "Global patent protection: channels of north and south welfare gain," J. Economic Dynamics and Control, Vol. 30, pp. 205-227, 2006.

[18] Kennedya, G. and Clarkb, D., "Outsourcing to China - Risks and benefits,"computer law \& security report, Vol 22, pp. 250-253, 2006.

[19] Yanlin, S. and Canhua, K., "Impact of Intellectual Property Protection on Foreign Direct Investment of Southern Firms," IEEE Symposium on Advanced Management of Information for Globalized Enterprises, pp. 229-233, 2008.

[20] Sang Ho Lee, "Personal portable device", International Patent, PCT / KR2007 / 001053, 2007.

[21] Sang Ho Lee, Bo Hyung Lee, Seong Ki Seo, "Slide type personal portable device", International Patent, PCT / WO2007 / 117080, 2007.

[22] Coello, C., Lamont, G. and Van Veldhuizen, D., Evolutionary Algorithms for Solving Multi-Objective Problems, Springer-Verlag, 2007.

[23] Goldberg, D., Genetic Algorithms in Search, Optimization, and Machine Learning, Addison-Wesley, 1989.

[24] Manning, C. and Schutze, H., Foundations of Statistical Natural Language Processing, MIT Press, 1999. 